\title{
High-density fluorescently labeled rolling-circle amplicons for DNA diagnostics
}

\author{
Irina V. Smolina ${ }^{a}$, Dmitry I. Cherny ${ }^{b, 1}$, Raymond M. Nietupski ${ }^{c}$, Thomas Beals ${ }^{c}$, \\ James H. Smith ${ }^{\text {c }}$, David J. Lane ${ }^{\mathrm{c}}$, Natalia E. Broude ${ }^{\mathrm{a}, *}$, Vadim V. Demidov ${ }^{\mathrm{a}, *}$ \\ ${ }^{a}$ Center for Advanced Biotechnology, Boston University, Boston, MA 02215, USA \\ ${ }^{\mathrm{b}}$ Max Planck Institute for Biophysical Chemistry, D-37077 Gottingen, Germany \\ ${ }^{\mathrm{c}}$ Hamilton Thorne Biosciences Inc., Beverly, MA 01915, USA
}

Received 15 March 2005

Available online 21 June 2005

Fluorescent labeling of nucleic acids with high density is very important for a wide area of DNA- and RNAbased diagnostics [1-5], but it still remains a challenge. Indeed, chemical synthesis of oligonucleotides with multiple fluorophores is an elaborate and expensive procedure [6-8]. Branched probes tagged with numerous fluorophores can alleviate this problem [2,9], but special complex assemblies are required in that case. Enzymatic synthesis of high-density labeled polynucleotides from fluorescently modified monomers by DNA polymerases is another alternative. However, despite some progress in this area, there are certain difficulties in enzymatically generating long fluorescent products using procedures based on primer extension [3,4,10-13].

Rolling-circle amplification (RCA), ${ }^{2}$ which is isothermal DNA polymerase-driven cyclic replication of small circular DNA probes, is often used as an efficient method to detect various analytes [14-16]. It was assumed that the RCA mechanism is different from that of primer-extension reactions using linear DNA templates. ${ }^{3}$ Based on that, we suggested that DNA polymerase during RCA might better tolerate fluorescently labeled deoxynucleotide triphosphates (dNTPs) and decided to investigate whether RCA can be employed for direct generation of densely labeled amplification products capable of intense fluorescence. ${ }^{4}$

We chose $\Phi 29$ DNA polymerase considering its wellknown robust RCA activity [23-25] and the hydrophilic dye Cy3 with which high-density labeling could be achieved without serious fluorescence quenching $[8,26] .^{5}$ All experiments were performed with $\mathrm{Cy} 3-\mathrm{dCTP}$ and $\mathrm{Cy} 3-\mathrm{dUTP}$, which substituted dCTP and dTTP, respectively, and with 88-nt DNA minicircle carrying Salmonella sequence marker [27].

Gel-electrophoretic analysis of RCA products obtained in solution with complete substitution of two pyrimidine dNTPs with Cy3-tagged fluorescent analogs showed that the efficiency of synthesis was reduced as compared to that with non-modified dNTPs Nevertheless,

\footnotetext{
* Corresponding authors. Fax: +617 3538501.

E-mail addresses: nebroude@bu.edu (N.E. Broude),vvd@bu.edu (V.V. Demidov).

${ }_{1}^{1}$ On leave from the Institute of Molecular Genetics, Moscow, Russia. Present address: Department of Biochemistry, Leicester University, Leicester LE1 7RH, UK.

2 Abbreviations used: RCA, rolling-circle amplification; dNTP, deoxynucleotide triphosphate; EM, electron microscopy; nt, nucleotide; bp, base pair; kb, kilo base (thousand base pairs).

${ }^{3}$ The shape of an RCA-active circular DNA probe is quite distinct from the normal DNA replicative form in that it is overall very strained and features abrupt tense boundaries [14,17]. This constrained configuration of replicating DNA minicircles is caused by their small size (typically $\leqslant 100 \mathrm{nt}[15])$ and by the stiffness of DNA duplexes formed as a result of primer annealing and extension along circular probes. The DNA duplexes are known to remain essentially straight up to 150 to $200 \mathrm{bp}$ [18-21]. Therefore, we hypothesized that this exceptional DNA structure, namely the "fold-back," sharp-edged DNA conformation at the site of DNA replication (see Fig.2A), might facilitate incorporation of fluorescently modified nucleotides by DNA polymerase.

${ }^{4}$ We are aware that a number of research groups have tried to use fluorescently modified dNTPs for direct labeling of RCA amplicons but gave up their attempts. Yet, the lack of any published data on this topic prompted us to perform our study to overcome disappointment in this rather straightforward and potentially useful diagnostic approach.

${ }^{5}$ In some cases, high-density labeling results in long but nonfluorescent reaction products due to self-quenching of closely located fluorophores [22].
} 
A
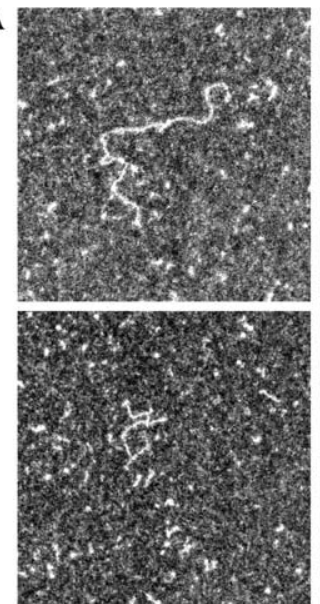

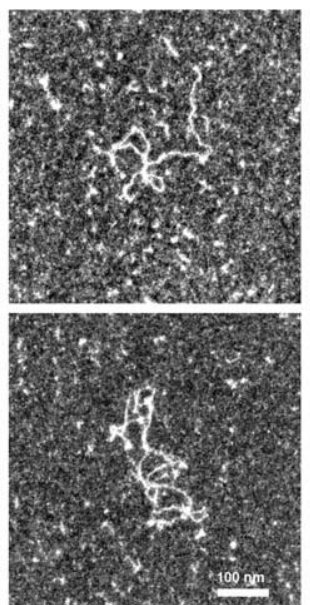

B

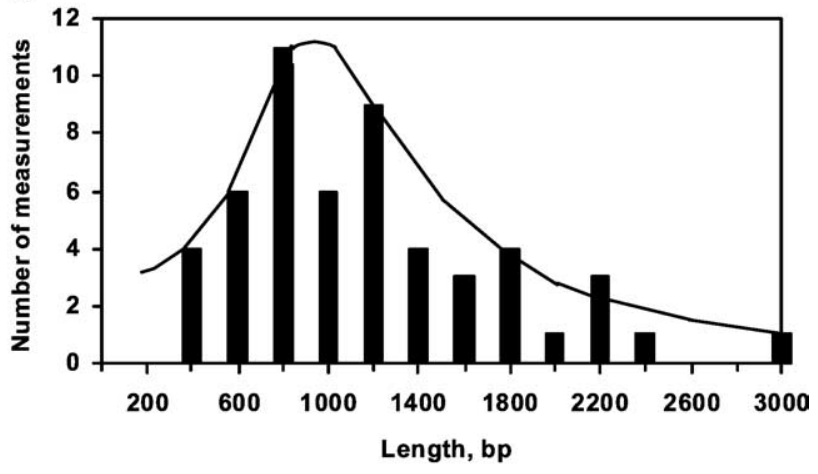

Fig. 1. EM visualization of Cy3-labeled RCA amplicons. (A) Images of exemplary RCA amplicons generated in solution with $100 \%$ Cy3-dCTP and Cy3-dUTP substitutions for corresponding dNTPs. (B) Histogram of lengths distribution for 53 RCA amplicons. A 5'-phosphorylated 88-nt circularized probe carrying Salmonella genome sequences at its termini, ${ }^{5} p$ GCGCCTTTCCAGACGCTTACCAAGAGCAACTACACG AATTCTC GATTAGGTTACTGCGATTAGCACAAGC GCTGTCACCCTGTATCGC, was circularized by ligation with Taq DNA ligase in the presence of biotin- ${ }^{5} \mathrm{~N}_{20}$ GGAAAGGCGCGCGATACAGG (splint), also employed as a primer in RCA. Complex of the DNA minicircle and primer (10 ${ }^{9}-10^{12}$ $\mathrm{mol}$ of the complex in $50 \mu \mathrm{l}$ ) was incubated at $37^{\circ} \mathrm{C}$ overnight with $1 \mathrm{U}$ of $\Phi 29$ DNA polymerase and $20 \mu \mathrm{M}$ dNTPs in the buffer containing $20 \mathrm{mM}$ Tris- $\mathrm{HCl}$ (pH 7.5), $10 \mathrm{mM} \mathrm{MgCl} 2,25 \mathrm{mM} \mathrm{NaCl}$, and $5 \mathrm{mM}$ dithiothreitol. dTTP and dCTP were completely substituted by their Cy3-fluorescent analogs. Gel-filtration purified RCA amplicons were annealed with the 88-nt complementary oligonucleotide for better visualization by EM. Samples for electron microscopy were prepared as described in Ref. [28]

the length of RCA amplicons reached 1 to $2 \mathrm{~kb}(10-20$ repeats) as compared with size standards (data not shown). Keeping in mind that multiply labeled DNA exhibits altered physicochemical properties, including anomalous gel-electrophoretic mobility $[10,12]$, we decided to directly measure the length of RCA products using electron microscopy (EM). EM visualization of Cy3-labeled RCA products (Fig. 1A) showed that the length of randomly chosen amplicons ranged from $400 \mathrm{bp}$ to $3 \mathrm{~kb}$ (Fig. 1B), with the average size of $1.2 \mathrm{~kb}$ being in agreement with initial gel-electrophoretic estimations.

Thus, these results mean that every RCA amplicon carries several hundred fluorophores, and the question is whether fluorescent signal is strong enough to be detected using fluorescence imaging instruments. To answer this question, we chose the surface-immobilized RCA format, shown schematically in Fig. 2A, that uses biotin-streptavidin interactions for primer immobilization. Based on the published data [23,29,30], we expected that RCA efficiency in heterogeneous systems would be close to that in a homogeneous solution.

Using conventional fluorescent microscopy, we detected RCA amplicons as the luminous dot-like objects, the number of which correlated with the amount of primers immobilized onto a surface but was substantially less than expected (Fig. 2B). We reasoned that when using fluorescent microscopy, we probably see only the brightest amplicons while the less intense and blurred signals are lost for analysis. To improve sensitivity and make assay applicable for automation, we employed the fluorescence imaging scanner, allowing the measurement of total fluorescence of RCA amplicons per specified area (Fig. 2C).
Using this approach, we detected as few as $10^{2}$ amplicons/ $\mathrm{mm}^{2}$ (Fig. 2D), corresponding to the detection limit of approximately thousand analyte molecules.

Thus, our study shows that direct incorporation of fluorescently labeled dNTPs during RCA allows synthesis of densely labeled RCA amplicons, which can be detected with high sensitivity using fluorescent scanning instruments. Although fluorescently modified dNTPs decrease considerably the degree of RCA amplification, high-density labeling compensates for that and the assay still allows detecting down to $10^{3}$ molecules of the analyte. With normal dNTPs, a much higher, usually more than $10^{3}$-fold, amplification can be achieved [24]. However, with postreplicative procedures it is difficult to reach high-density labeling. Indeed, our studies of RCA reactions with normal dNTPs and postreplicative labeling of amplicons using dual-labeled hybridization probes demonstrated somewhat better, but comparable, sensitivity (Fig. 2D). When considering advantages of the proposed approach, we should note that the surface-immobilized format with direct high-density fluorescent labeling is well suited to high-throughput parallel analysis on diagnostic arrays. ${ }^{6}$ If single-molecule sensitivity

\footnotetext{
${ }^{6}$ In fact, no extra probes or extra steps are required. On the contrary, traditional hybridization approaches require rather expensive "decorator" probes, hybridization/washing steps, and amplicon-collapsing proteins for greater brightness [23,31]. Note that in our case RCA amplicons are single-stranded and thus are highly compact by themselves, as compared with partly double-stranded (stretched) probe-hybridized amplicons; therefore, there is no need to enhance their brightness. Another positive feature of our approach is that we measure total signal with the fluorescence-integrating device (scanner), which is amenable to automation and is less subjective than measuring single bright objects [23,31].
} 
A

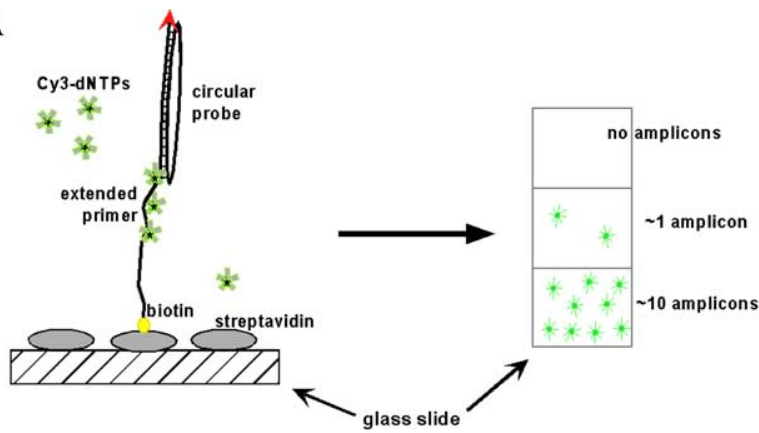

B
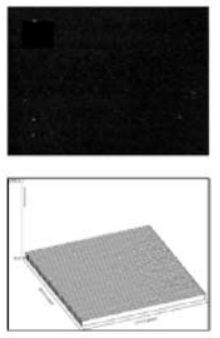
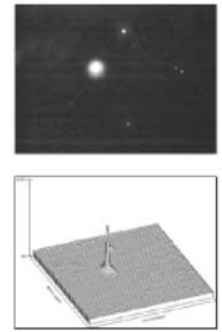
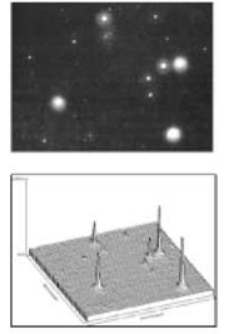

C

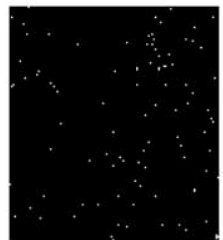

$10 \mathrm{~mol} / \mathrm{mm}^{2}$

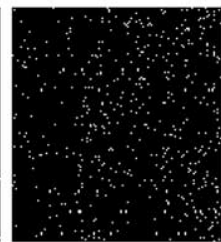

$10^{2} \mathrm{~mol} / \mathrm{mm}^{2}$

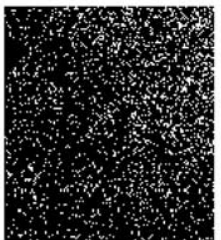

$10^{3} \mathrm{~mol} / \mathrm{mm}^{2}$

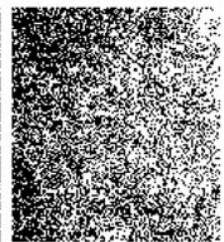

$10^{4} \mathrm{~mol} / \mathrm{mm}^{2}$

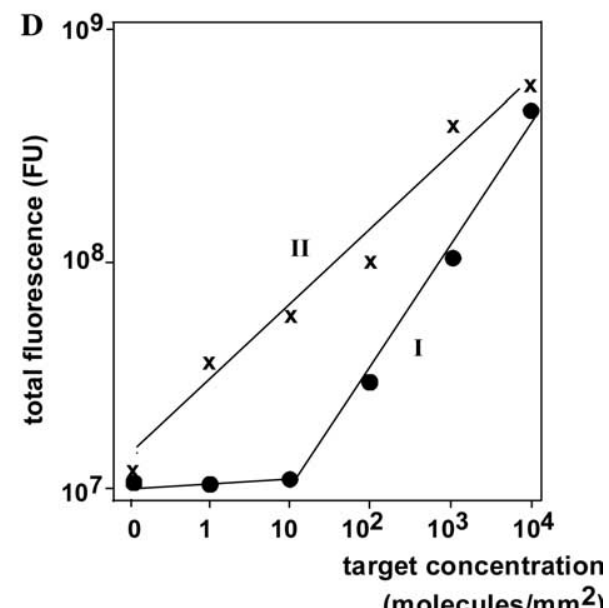

(molecules $/ \mathrm{mm}^{2}$ )

Fig. 2. Fluorescent microscopy of surface-immobilized fluorogenic RCA amplicons. (A) Schematics of the RCA experiments. Note the unusual structure of the rolling circle complex ${ }^{3}$. (B) Detection of RCA amplicons with fluorescent microscope. A specified amount (10 $\mu$ l) of the DNA minicircle complex with the primer was spread onto approximately $1 \mathrm{~cm}^{2}$ area of streptavidin-covered glass slides $\left(10^{6}\right.$ streptavidin molecules/pixel). After a few minutes, $40 \mu \mathrm{l}$ of solution with all other reaction components was added. dTTP and dCTP were completely substituted by their Cy3-fluorescent analogs. The spot was covered with the thin cover glass and fixed with glue (rubber cement). The samples were incubated at $37^{\circ} \mathrm{C}$ overnight; washed by successively immersing the slides in (i) $2 \times \mathrm{SSC}$ with $2 \%$ sodium dodecyl sulfate, (ii) $2 \times \mathrm{SSC}$ with $50 \%$ formamide, (iii) $2 \times \mathrm{SSC}$, and (iv) $0.2 \times \mathrm{SSC}$; dried in air; and subjected to microscopy analysis with a Nikon E800 fluorescent microscope $(\times 20)$ (upper), and images were processed by Image J software (lower). Left to right: "no circle" control (background fluorescence), approximately $100 \mathrm{amplicons} / \mathrm{mm}^{2}$, and approximately $1000 \mathrm{amplicons} / \mathrm{mm}^{2}$. (C) GenePix scanner images of the same slides $\left(10 \mu \mathrm{m}\right.$ resolution, ImageJ software). Images with 10 amplicons $/ \mathrm{mm}^{2}$ look similar to the background images (not shown). (D) Total fluorescence measured as a function of target load for RCA reactions with 100\% Cy3-dCTP/Cy3-dUTP in the dNTP mixture (I) or for RCA reactions with all normal dNTPs and with the use of detector oligonucleotide tagged with Cy3 at both of its termini (II). Areas of $10 \times 10 \mathrm{~mm}\left(10^{6}\right.$ pixels $)$ were analyzed using SigmaPlot software. Pixels with fluorescence less that $200 \mathrm{FU}$ were considered as background and were not counted.

is not an issue, direct RCA labeling and automated scanning of the arrays could be an assay of choice.

In summary, we have reported that RCA with $\Phi 29$ DNA polymerase and Cy3-labeled dNTPs is a simple tool for generation of high-density fluorescently labeled amplicons. When biotinylated RCA primer is immobilized on streptavidin-covered glass slides, the assay employing fluorescent scanners can be used for direct and sensitive quantitation of corresponding analytes without any hybridization probes.

\section{Acknowledgments}

We thank Charles Lee for access to the fluorescent scanner, Charles R. Cantor for comments on the manuscript, and Harry McCoy and Diarmaid H. Douglas-
Hamilton for participating in discussions. This study was supported by a sponsored research agreement between Boston University and Hamilton Thorne Biosciences Inc. (Charles Cantor, PI).

\section{References}

[1] J. Zimmermann, W. Ludwig, K.H. Schleifer, DNA polynucleotide probes generated from representatives of the genus Acinetobacter and their application in fluorescence in situ hybridization of environmental samples, Syst. Appl. Microbiol. 24 (2001) 238-244.

[2] E. Manduchi, L.M. Scearce, J.E. Brestelli, G.R. Grant, K.H. Kaestner, C.J. Stoeckert Jr., Comparison of different labeling methods for two-channel high-density microarray experiments, Physiol. Genom. 10 (2002) 169-179.

[3] G. Giller, T. Tasara, B. Angerer, K. Muhlegger, M. Amacker, H. Winter, Incorporation of reporter molecule-labeled nucleotides by DNA polymerases: I. Chemical synthesis of various reporter 
group-labeled 2'-deoxyribonucleoside-5'-triphosphates, Nucleic Acids Res. 31 (2003) 2630-2635.

[4] T. Tasara, B. Angerer, M. Damond, H. Winter, S. Dorhofer, U. Hubscher, M. Amacker, Incorporation of reporter moleculelabeled nucleotides by DNA polymerases: II. High-density labeling of natural DNA, Nucleic Acids Res. 31 (2003) 2636-2646.

[5] A. Richter, C. Schwager, S. Hentze, W. Ansorge, M.W. Hentze, M. Muckenthaler, Comparison of fluorescent tag DNA labeling methods used for expression analysis by DNA microarrays, BioTechniques 33 (2002) 620-630.

[6] J. Haralambidis, M. Chai, G.W. Tregear, Preparation of basemodified nucleosides suitable for non-radioactive label attachment and their incorporation into synthetic oligodeoxyribonucleotides, Nucleic Acids Res. 15 (1987) 4857-4876.

[7] D. Singh, V. Kumar, K.N. Ganesh, Oligonucleotides: V. Synthesis and fluorescence studies of DNA oligomers d(AT) 5 containing adenines covalently linked at C-8 with dansyl fluorophore, Nucleic Acids Res. 18 (1990) 3339-3345.

[8] J.B. Randolph, A.S. Waggoner, Stability, specificity, and fluorescence brightness of multiply-labeled fluorescent DNA probes, Nucleic Acids Res. 25 (1997) 2923-2929.

[9] R.L. Stears, R.C. Getts, S.R. Gullans, A novel, sensitive detection system for high-density microarrays using dendrimer technology, Physiol. Genom. 3 (2000) 93-99.

[10] Z. Zhu, J. Chao, H. Yu, A.S. Waggoner, Directly labeled DNA probes using fluorescent nucleotides with different length linkers, Nucleic Acids Res. 22 (1994) 3418-3422.

[11] Z. Zhu, A.S. Waggoner, Molecular mechanism controlling the incorporation of fluorescent nucleotides into DNA by PCR, Cytometry 28 (1997) 206-211.

[12] M.A. Augustin, W. Ankenbauer, B. Angerer, Progress towards single-molecule sequencing: Enzymatic synthesis of nucleotide-specifically labeled DNA, J. Biotechnol. 86 (2001) 289-301.

[13] J.P. Anderson, B. Angerer, L.A. Loeb, Incorporation of reporterlabeled nucleotides by DNA polymerases, BioTechniques 38 (2005) 257-264.

[14] V.V. Demidov, Rolling-circle amplification in DNA diagnostics: The power of simplicity, Expert Rev. Mol. Diagn. 2 (2002) 542-548.

[15] D.Y. Zhang, B. Liu, Detection of target nucleic acids and proteins by amplification of circularizable probes, Expert Rev. Mol. Diagn. 3 (2003) 237-248.

[16] S. Bakht, X. Qi, Ligation-mediated rolling-circle amplificationbased approaches to single nucleotide polymorphism detection, Expert Rev. Mol. Diagn. 5 (2005) 111-116.

[17] M. Frieden, E. Pedroso, E.T. Kool, Tightening the belt on polymerases: Evaluating the physical constraints on enzyme substrate size, Angew. Chem. Intl. Ed. 38 (1999) 3654-3657.
[18] D. Shore, J. Langowski, R.L. Baldwin, DNA flexibility studied by covalent closure of short fragments into circles, Proc. Natl. Acad. Sci. USA 78 (1981) 4833-4837.

[19] L. Ulanovsky, M. Bodner, E.N. Trifonov, M. Choder, Curved DNA: Design, synthesis, and circularization, Proc. Natl. Acad. Sci. USA 83 (1986) 862-866.

[20] P.J. Hagerman, Flexibility of DNA, Annu. Rev. Biophys. Biophys. Chem. 17 (1988) 265-286.

[21] H. Kuhn, D.I. Cherny, V.V. Demidov, M.D. Frank-Kamenetskii, Inducing and modulating anisotropic DNA bends by pseudocomplementary peptide nucleic acids, Proc. Natl. Acad. Sci. USA 101 (2004) 7548-7553.

[22] S. Brakmann, S. Löbermann, High-density labeling of DNA: Preparation and characterization of the target material for singlemolecule sequencing, Angew. Chem. Intl. Ed. 40 (2001) 1427-1429.

[23] P.M. Lizardi, X. Huang, Z. Zhu, P. Bray-Ward, D.C. Thomas, D.C. Ward, Mutation detection and single-molecule counting using isothermal rolling-circle amplification, Nat. Genet. 19 (1998) 225-232.

[24] J. Baner, M. Nilsson, M. Mendel-Hartvig, U. Landegren, Signal amplification of padlock probes by rolling circle replication, Nucleic Acids Res. 26 (1998) 5073-5078.

[25] M. Salas, M. de Vega, J.M. Lázaro, L. Blanco, Phi29 DNA polymerase, a potent amplification enzyme, in: V.V. Demidov, N.E. Broude (Eds.), DNA Amplification: Current Technologies and Applications, Horizon Bioscience, Wymondham, UK, 2004, pp. 21-34.

[26] H. Yu, J. Chao, D. Patek, R. Mujumdar, S. Mujumdar, A.S. Waggoner, Cyanine dye dUTP analogs for enzymatic labeling of DNA probes, Nucleic Acids Res. 22 (1994) 3226-3232.

[27] R.M. Nietupski, S.G. Wilson, J. Shah, S.W. Chan, D.N. Halbert, D.J. Lane, Probes and methods for the detection of Salmonella, U.S. patent 5,147,778 (1992).

[28] D.I. Cherny, T.M. Jovin, Electron and scanning force microscopy studies of alterations in supercoiled DNA tertiary structure, J. Mol. Biol. 313 (2001) 295-307.

[29] C.R. Sabanayagam, C. Berkey, U. Lavi, C.R. Cantor, C.L. Smith, Molecular DNA switches and DNA chips, in: M. Ferrari (Ed.), Micro- and Nanofabricated Structures and Devices for Biomedical and Environmental Applications, vol. 3606, International Society for Optical Engineering, San Jose, CA, 1999, pp. 90-97.

[30] A. Hatch, T. Sano, J. Misasi, C.L. Smith, Rolling circle amplification of DNA immobilized on solid surfaces and its application to multiplex mutation detection, Genet. Anal. 15 (1999) $35-40$.

[31] X.B. Zhong, P.M. Lizardi, X.H. Huang, P.L. Bray-Ward, D.C. Ward, Visualization of oligonucleotide probes and point mutations in interphase nuclei and DNA fibers using rolling circle DNA amplification, Proc. Natl. Acad. Sci. USA 98 (2001) 3940-3945. 